\title{
BMJ Open Behavioural activation in nursing homes to treat depression (BAN-Dep): study protocol for a pragmatic randomised controlled trial
}

\author{
Diana Velasquez Reyes, ${ }^{1}$ Hema Patel, ${ }^{2}$ Nicola Lautenschlager, ${ }^{1}$ Andrew H Ford, ${ }^{2}$ \\ Eleanor Curran, ${ }^{1}$ Rachael Kelly, ${ }^{2}$ Rhoda Lai, ${ }^{1}$ Terence Chong (1) , ${ }^{1}$ Leon Flicker, ${ }^{2}$ \\ David Ekers, ${ }^{3}$ Simon Gilbody, ${ }^{3}$ Christopher Etherton-Beer, ${ }^{2}$ Dina Lo Giudice, ${ }^{4}$ \\ Kathryn A Ellis, ${ }^{1}$ Angelita Martini, ${ }^{5}$ Osvaldo P Almeida (i) ${ }^{2}$
}

To cite: Velasquez Reyes D,

Patel H, Lautenschlager N, et al. Behavioural activation in nursing homes to treat depression (BAN-Dep): study protocol for a pragmatic randomised controlled trial. BMJ Open 2019;9:e032421. doi:10.1136/ bmjopen-2019-032421

- Prepublication history for this paper is available online. To view these files, please visit the journal online (http://dx.doi org/10.1136/bmjopen-2019032421).

Received 18 June 2019 Revised 06 August 2019 Accepted 11 October 2019

Check for updates

(c) Author(s) (or their employer(s)) 2019. Re-use permitted under CC BY-NC. No commercial re-use. See rights and permissions. Published by BMJ.

${ }^{1}$ Psychiatry, The University of Melbourne, Melbourne, Victoria, Australia

${ }^{2}$ Medical School, University of Western Australia Faculty of Medicine Dentistry and Health Sciences, Perth, Western

Australia, Australia

${ }^{3}$ Psychiatry, University of York, York, UK

${ }^{4}$ Aged Care, Melbourne Health, Parkville, Victoria, Australia

${ }^{5}$ Brightwater Care Group,

Osborne Park, Western Australia, Australia

Correspondence to

Professor Osvaldo P Almeida; osvaldo.almeida@uwa.edu.au

\section{ABSTRACT}

Introduction Depression is a common disorder among older people living in residential aged care facilities. Several trials have demonstrated the effectiveness of behavioural therapies in treating depressive symptoms in older adults living in the community and in residential aged care. Behavioural Activation is demonstrably effective even when delivered by non-specialists (staff without formal psychological training), although strategies for adapting its use in residential aged care facilities are yet to be explored. This study will determine whether training residential care staff in the use of a structured Behavioural Activation programme is more effective at decreasing depressive symptoms among older residents than internet-based training about depression recognition and management alone.

Method and analysis The behavioural activation in nursing homes to treat depression (BAN-Dep) trial is a pragmatic two-arm parallel clustered randomised controlled trial. It will recruit 666 residents aged 60 or older from 100 residential aged care facilities, which will be randomly assigned to the Behavioural Activation or control intervention. Staff in both treatment groups will be encouraged to complete the Beyondblue Professional Education to Aged Care e-learning programme to improve their recognition of and ability to respond to depression in older adults. Selected staff from intervention facilities will undergo additional training to deliver an 8-module Behavioural Activation programme to residents with subthreshold symptoms of depression-they will receive ongoing Mental support from trained Behavioural Activation therapists. Outcome measures will be collected by blind research officer at baseline and after 3, 6 and 12 months. The Patient Health Questionnaire-9 is the primary outcome measure of the study.

Ethics and dissemination The trial will comply with the principles of the Declaration of Helsinki for Human Rights and is overseen by the University of Western Australia (reference RA/4/20/4234) and Melbourne Health (reference number HREC/18/MH/47) Ethics Committees. The results of this research project will be disseminated through publications and/or presentations in a variety of media to health professionals, academics, clinicians and the public. Only de-identified group data will be presented.

\section{Strengths and limitations of this study}

- The behavioural activation in nursing homes to treat depression (BAN-Dep) pragmatic randomised controlled trial will collect and compare long term followup data after the application of the intervention.

- The BAN-Dep trial Behavioural Activation intervention integrates aspects of collaborative care approach to facilitate the successful delivery of the intervention allowing a rapid scale-up and ensuring its sustainability over time.

- The BAN-Dep pragmatic approach provides practical education of staff, both for the intervention and control arm.

- The study is limited to the aged care facilities of two Australian cities and will exclude people with moderate to severe cognitive impairment.

Trial registration ACTRN12618000634279.

\section{INTRODUCTION}

Depression is a disorder that affects about $8 \%$ of older Australians living in the community. ${ }^{12}$ While various demographic, lifestyle and clinical factors may contribute to increase the risk of depression in later life, increased frailty and functional decline are robust predictors of depression in the very old (75 years or over),,$^{3-5}$ and also predict transition to living in residential aged care facilities, contributing to substantially higher prevalence of depression in residential aged care facilities compared with the community. ${ }^{67} \mathrm{~A}$ survey of 22 residential aged care facilities in Perth, Australia showed that clinically significant symptoms of depression were present in $50 \%$ of newly admitted residents (Geriatric Depression Scale score $\geq 5 / 15) .{ }^{8}$ Notably, a systematic review found the median prevalence of major depressive disorder and of 
clinically significant depressive symptoms in residential aged care facilities to be $10 \%$ and $30 \%$, respectively. ${ }^{7}$

Despite its high prevalence, depression remains both under-recognised and poorly treated in residential aged care facilities. ${ }^{8-10}$ Atypical presentations of depressive symptoms in older adults and those with cognitive impairment compared with younger adults, limited access to relevant information, presence of multiple concurrent morbidities, social stereotypes and workload constraints have been identified as barriers to recognition and treatment. ${ }^{11}$ However, randomised controlled trials of strategies for increasing the recognition of depression, such as assertive case identification using mental health screening and early referrals to psychogeriatric services, have not appeared to result in meaningful improvement in the mental health outcomes of residents. ${ }^{8}$ Staff training alone has been found to be insufficient to increase the correct identification of residents with depression-while the use of a screening protocol and training led to staff identifying more depressed residents, rates of misclassification (ie, incorrectly identifying non-depressed residents as depressed) increased. ${ }^{10}$

Treating depression in older adults is challenging due to the relative paucity of guidelines specific to this age group. ${ }^{12}$ Antidepressant medications remain the mainstay of treatment for depression in older people, despite current clinical treatment guidelines recommending a more holistic approach, particularly for subsyndromal, mild or moderate depression. ${ }^{12-14}$ Antidepressant prescription rates are rising, with one review of prescribing data from 12556 American residential aged care facilities reporting that prescriptions more than doubled between 1996 and 2006. ${ }^{15}$ A cross-sectional retrospective cohort study of residents from 150 residential aged care facilities in Australia reported nearly two-thirds (61.2\%) were taking an antipsychotic, anxiolytic/hypnotic and/or antidepressant medication on a regular basis. ${ }^{16}$ When "pro re nata (PRN)' (as needed) use was taken into account, more than half of the residents $(54.1 \%)$ were taking antipsychotic and/or benzodiazepine agents. ${ }^{16}$ This has occurred despite limited evidence that antidepressant use is effective in older people, especially among those with dementia. ${ }^{16-19}$ A review of the effectiveness of antidepressants for older adults in residential aged care facilities with Major Depressive Disorder or minor depression identified 11 studies, of which four were randomised trials. ${ }^{20}$ Of these, only two small studies ( 55 participants in total) had a suitable placebo-control group, and neither showed a significant benefit associated with the use of antidepressants. ${ }^{20}$ Further, the risk of polypharmacy and harmful drug interactions are significant concerns when treating older adults' depressive symptoms pharmacologically because individuals this age group can be more susceptible to serious adverse effects such as daytime drowsiness, dizziness, falls, hyponatraemia and stroke. ${ }^{921-23}$

Non-pharmacological interventions may have an important and under-recognised role in the treatment of depression in later life, and have been shown to have effect sizes that are comparable to those of antidepressants. ${ }^{12}{ }^{24}$ Specific interventions include behavioural therapies, cognitive behavioural therapies (CBT), third wave CBT, psychodynamic therapies, humanistic therapies (eg, existential therapy and non-directive therapies), integrative therapies, systemic therapies and reminiscence therapies. ${ }^{25}$ Behavioural therapies aim to change maladaptive behaviours in order to treat mental health problems, and are promising as treatments for depressive symptoms in older adults as they tend to be simpler and more costeffective than other psychological therapies without having the adverse effects commonly associated with medication use. $^{24-29}$

Behavioural Activation is a type of behavioural therapy in which individuals learn to monitor their mood and daily activities and to understand the connection between them. ${ }^{30}$ By increasing participation in rewarding activities and reducing avoidance behaviours, Behavioural Activation aims to increase exposure to sources of positive reinforcement that are missed when depressed individuals maladaptively withdraw, leading to symptom improvement. ${ }^{30} 31$ A systematic review and meta-analysis of Behavioural Activation as a treatment for depression (where 4 of the 16 included studies were conducted with older adults and from these only one included participants with dementia) concluded that Behavioural Activation was as effective as CBT and particularly attractive as a treatment for challenging populations because its relative simplicity enables better patient understanding. ${ }^{28} \mathrm{~A}$ large randomised controlled trial comparing Behavioural Activation and CBT confirmed these findings and showed that Behavioural Activation can be delivered by mental health workers without formal psychological training, which contributes to reducing its cost. ${ }^{26}$

Importantly, evidence from randomised controlled trials suggests that Behavioural Activation is well accepted by participants with depressive symptoms. A trial randomised 241 adults with Major Depressive Disorder to treatment with Behavioural Activation, cognitive therapy, paroxetine or placebo and found that the efficacy of Behavioural Activation and paroxetine were similar and more effective than cognitive therapy and placebo. ${ }^{29}$ Further, retention during the initial 8 weeks of the trial was higher for people treated with Behavioural Activation $(91 \%)$ than paroxetine (64\%). ${ }^{29}$ Another trial has demonstrated that intense, frequent sessions of Behavioural Activation are not needed for older adults who required home care, who experienced significant improvements in their depressive symptoms and quality of life after a total of 6 sessions at monthly intervals of Behavioural Activation. ${ }^{32}$ These Behavioural Activation sessions were delivered within a larger intervention that included medication reviews and referrals, suggesting that Behavioural Activation interventions could be successfully incorporated within existing care frameworks. ${ }^{32}$ In this trial $31 \%$ participants had dementia.

Behavioural Activation, either alone or as part of a multimodal intervention for depression, is currently 
being studied in residential aged care facilities settings. Behavioural Activation formed a key component of a multidisciplinary care intervention in a Dutch steppedwedge cluster-randomised controlled trial that led to a reduction in the prevalence of depression among older people in residential aged care facilities, although the benefit was limited to residents without significant cognitive impairment. ${ }^{33}$ In another randomised controlled trial, BE-ACTIV, 23 American residential aged care facilities were randomly assigned to usual care or a 10-session Behavioural Activation intervention conducted by trained therapists. ${ }^{34}$ A larger proportion of participants in intervention facilities than those in usual care facilities experienced remission of symptoms after 3 months, but some of these gains were lost by 6 months. ${ }^{34}$ The participants in this study were eligible only with a Mini-Mental State Examination (MMSE) score above 14. The authors of that study queried whether the delivery of Behavioural Activation by external 'experts' may have contributed to the lack of sustainability in benefits seen. In 2017 researchers undertook a systematic review analysing the effect that Behavioural Activation has on depressive symptoms in older people living in the community and in longterm care settings. ${ }^{35}$ In this review the authors identified six randomised control trials in residential aged care settings. From these trials two included only participants with dementia, the other had different MMSE scores as inclusion criteria. The authors reported favourable results towards behavioural activation therapies in older adults living in the community (SMD $=-0.72,95 \% \mathrm{CI}-1.04$ to -0.41 , efficacy at 4-12 weeks) but found no difference between the intervention and treatment as usual groups in long term care settings $(\mathrm{SMD}=-0.43,95 \% \mathrm{CI}-0.87$ to 0.01 , efficacy at $6-12$ weeks $\left.\mathrm{I}^{2}=65 \%\right) .{ }^{35}$

More recently, researchers have investigated Behavioural Activation within models of care that use non-specialist (staff without formal psychological training) for delivery. Given the frequency and prevalence of depressive symptoms in older populations, reliance on specialist staff may significantly limit the real-world impact of novel treatment strategies. The CASPER trial (ISRCTN02202951) investigated whether a Behavioural Activation programme, delivered by case managers with support available from psychiatrists or physicians where required, could prevent the onset of clinically significant depression among 344 older adults with subthreshold symptoms at baseline. ${ }^{36}$ This randomised controlled trial showed that Behavioural Activation decreased participants' relative risk of developing clinically significant symptoms of depression and reduced the severity of their depressive and anxiety symptoms, with these benefits remaining at 12-month follow-up. ${ }^{36}$ Further, in the Cost and Outcome of BehavioRal Activation (COBRA) trial, Behavioural Activation delivered by mental health workers without formal psychological training who received 5 days' training and regular supervision was shown to be comparable to CBT, and was significantly less costly to fund than the professional CBT therapists. ${ }^{26}$ The duration of the Behavioural Activation sessions in the COBRA trial was about 1 hour, with 20 sessions delivered over 16 weeks, plus four additional booster sessions if required. ${ }^{26}$ In contrast, in the CASPER trial, which was targeted towards older adults with long-term physical health problems, Behavioural Activation sessions were much shorter, averaging half an hour each, and participants received an average of six sessions in a mix of face-to-face and telephone modes of delivery. ${ }^{36} \mathrm{In}$ residential aged care facilities, where many residents would be frailer, evidence that shorter, less intense sessions delivered by non-specialist (staff without formal psychological training) can still be effective is extremely valuable.

In planning successful interventions in residential aged care facilities settings, it is important to note that research focusing on dementia has demonstrated the importance of supported local leadership ('Dementia Champions') to promote and sustain the acquisition of practical knowledge about dementia assessment and care. ${ }^{37}$ While studies of interventions for depression in residential aged care facilities to date have not yet adopted similar models, it is plausible that using local staff as 'Mental Health Champions' to deliver Behavioural Activation to residents could further help to sustain the benefits of a Behavioural Activation programme, while also providing useful skills and professional development for staff. ${ }^{37}$

Overall, research in this area to date has shown that improved recognition of depression in residential aged care facilities is an important step but on its own has limited impact on the clinical outcome of residents over time, and that non-pharmacological interventions may be an under-recognised but important treatment direction for this vulnerable population. Interventions that include a Behavioural Activation component show promise given the evidence of their effectiveness in treating depressive symptoms and preventing the onset of Major Depressive Disorder in older adults, as well as being cost-effective and practical. However, there are only a small number of trials supporting this, and the most effective form of delivery for enhancing the sustainability of symptom improvements remains unclear. To address this gap in the literature, this study seeks to trial the use of local 'Mental Health Champions' to lead a Behavioural Activation intervention for depression in residential aged care facilities. This approach to delivering Behavioural Activation in residential aged care facilities is novel, but builds on current knowledge that non-specialist (staff without formal psychological training) can be trained to deliver Behavioural Activation with effective outcomes and, hopefully, contribute to sustained gains over time. Furthermore, this study will add to the currently limited knowledge about the effectiveness of Behavioural Activation in residential aged care facilities.

\section{Aims and hypotheses}

The aim of the present trial is to investigate whether training local residential aged care facilities staff members to deliver a structured Behavioural Activation programme can decrease depressive symptoms among older adults living in residential aged care facilities. 
The Behavioural Activation programme will be based on Pasterfield and colleagues' Behavioural Activation manual, which was adapted for the needs and considerations for older adults, and was used in the CASPER trial to produce significant improvements in communitydwelling older adults' depressive symptoms. ${ }^{36} 38$ This trial will assess whether the Behavioural Activation intervention is more effective in treating depressive symptoms than general staff training using a currently available e-learning tool, the Beyondblue e-learning Professional Education to Aged Care (PEAC) programme. Beyondblue is an Australian organisation whose mission is to improve knowledge of and skills in managing mental health in the community and in work and educational settings by providing support and educational tools. The PEAC was designed to educate staff to recognise and manage symptoms of depression and anxiety in older adults living in residential aged care facilities. ${ }^{39}$ The cost effectiveness of delivering the Behavioural Activation intervention in terms of cost per quality adjusted life year gained by the intervention will also be investigated.

It is hypothesised that older adults living in residential aged care facilities assigned to the Behavioural Activation intervention arm will have lower depression scores as measured by the Patient Health Questionnaire (PHQ-9) after the 3-month intervention period than their counterparts living in control (PEAC only) residential aged care facilities, and that this difference will be sustained after 6 and 12 months. Further, it is hypothesised that a lower proportion of older adults living in residential aged care facilities assigned to the Behavioural Activation intervention arm will show clinically significant symptoms of depression than their counterparts living in control residential aged care facilities after the intervention period and at 6-month and 12-month follow-up.

Secondarily, it is hypothesised that older adults living in Behavioural Activation intervention residential aged care facilities will have lower anxiety and higher quality of life scores, and greater knowledge about depression than those living in control residential aged care facilities at the 3-month, 6-month and 12-month timepoints. Finally, it is hypothesised that Mental Health Champions working in Behavioural Activation intervention residential aged care facilities will have greater knowledge about depression than those working in control residential aged care facilities at the 3-month, 6-month and 12-month timepoints.

\section{METHODS AND ANALYSIS \\ Design}

The BAN-Dep trial is a two-arm, parallel, clustered, pragmatic randomised controlled trial investigating whether training local staff members to deliver a structured Behavioural Activation programme enhances the benefits of the Beyondblue PEAC e-learning programme and decreases the prevalence of depression among older adults living in residential aged care facilities. The study assessments and study periods are shown in figure 1 .
There will be four measurement points in both intervention and control residential aged care facilities: baseline, and 3, 6 and 12 months postintervention.

\section{Participants}

The BAN-Dep trial will first recruit residential aged care facilities in the metropolitan regions of Perth and Melbourne, Australia. Facility managers will be approached about participating in the trial and provide consent on behalf of their facility to participate. Participating residential aged care facilities will be asked to nominate one or two staff members to take the role of Mental Health Champions, who will who encourage other facility staff to complete the PEAC, deliver the Behavioural Activation intervention to the residents included in the study (in residential aged care facilities randomised to the intervention arm) and facilitate project-related activities. Mental Health Champions can be any clinical or care staff member who has regular contact with residents. Residents from the participating residential aged care facilities who the Mental Health Champions and facility managers nominate as potentially eligible will then be approached to participate in the study.

Eligibility criteria for residential aged care facilities' residents Permanent residents of participating residential aged care facilities who are aged 60 years or over and report having depressive symptoms will be eligible to participate in the BAN-Dep trial. The presence of reported depressive symptoms will be determined by an affirmative answer to at least one of the following questions: (1) over the past month, have you often been bothered by feeling down, depressed or hopeless? (2) Over the past month, have you often been bothered by having little interest or pleasure in doing things? The use of these two questions, commonly known as the Whooley questions, has been shown to be a valid and quick case-finding instrument for detecting depression in primary care $(96 \%$ sensitivity and $57 \%$ specificity). ${ }^{40}$

We will exclude residents who:

1. Have a MMSE score lower than 18 (moderate to severe cognitive impairment) ${ }^{41}$

2. Have a disorder that impedes effective communication (eg, severe sensory impairment),

3. Have a physical illness that would preclude participation in the research activities, for example, severe sensory deficits.

4. Have active psychotic symptoms or suicidal ideation.

5. Have a PHQ-9 score $<5$ (ie, no or minimal depressive symptoms). ${ }^{42}$

6. Have difficulty communicating effectively in English, or

7. Are unable to provide informed consent to participate.

\section{Intervention}

The intervention will consist of two main components. The first component, the PEAC e-learning package from Beyondblue, will be delivered to all participating residential 


\begin{tabular}{|c|c|c|c|c|c|c|}
\hline TIMEPOINT & $\begin{array}{c}\text { Pre } \\
\text { intervention }\end{array}$ & Baseline & Intervention & $\begin{array}{c}\text { Post } \\
\text { intervention } \\
\text { Week } 12 \\
\text { follow up }\end{array}$ & $\begin{array}{c}\text { Post } \\
\text { intervention } \\
\text { Week } 26 \\
\text { follow up }\end{array}$ & $\begin{array}{c}\text { Post } \\
\text { intervention } \\
\text { Week } 52 \text { follow } \\
\text { up }\end{array}$ \\
\hline \multicolumn{7}{|l|}{ ENROLMENT: } \\
\hline \multirow{4}{*}{$\begin{array}{l}\text { Eligibility screen } \\
\text { Informed consent } \\
\text { Allocation } \\
\text { Baseline assessment }\end{array}$} & $X$ & & & & & \\
\hline & $\mathrm{X}$ & & & & & \\
\hline & $X$ & & & & & \\
\hline & & $X$ & & & & \\
\hline \multicolumn{7}{|l|}{ INTERVENTIONS: } \\
\hline \multicolumn{7}{|l|}{$\begin{array}{l}\text { e-learning program from Beyondblue: } \\
\text { Professional Education to Aged Care }\end{array}$} \\
\hline $\begin{array}{l}\text { 8-step behavioural activation (BA) } \\
\text { program training and delivery }\end{array}$ & & & & & & \\
\hline \multicolumn{7}{|l|}{ ASSESSMENTS: } \\
\hline Demographic Data & & $\mathrm{X}$ & & & & \\
\hline Interests and hobbies & & $\mathrm{X}$ & & $\mathrm{X}$ & $\mathrm{X}$ & $\mathrm{X}$ \\
\hline PHQ-9: Patient Health Questionnaire-9 & & $\mathrm{X}$ & & $\mathrm{X}$ & $\mathrm{X}$ & $\mathrm{X}$ \\
\hline Severity of Depressive Symptoms & & $\mathrm{X}$ & & $x$ & $\mathrm{X}$ & $x$ \\
\hline Generalized Anxiety Disorder-7GAD-7 & & $\mathrm{X}$ & & $\mathrm{X}$ & $\mathrm{X}$ & $x$ \\
\hline $\begin{array}{l}\text { Knowledge of Late Life Depression Scale } \\
\text { (KLLD) }\end{array}$ & & $\mathrm{X}$ & & $X$ & $\mathrm{X}$ & $X$ \\
\hline Slips, falls, injuries and fractured bones & & $\mathrm{X}$ & & $X$ & $\mathrm{X}$ & $X$ \\
\hline General Health SF-12 & & $\mathrm{X}$ & & $\mathrm{x}$ & $\mathrm{X}$ & $X$ \\
\hline De Jong Gierveld Loneliness Scale & & $x$ & & $\mathrm{X}$ & $\mathrm{X}$ & $x$ \\
\hline Lubben Social Network Scale & & $\mathrm{X}$ & & $\mathrm{X}$ & $\mathrm{X}$ & $\mathrm{X}$ \\
\hline Smoking History & & $\mathrm{X}$ & & $\mathrm{X}$ & $\mathrm{X}$ & $\mathrm{X}$ \\
\hline Medical History & & $\mathrm{X}$ & & $\mathrm{X}$ & $\mathrm{X}$ & $\mathrm{x}$ \\
\hline Modified Barthel Index & & $\mathrm{X}$ & & $\mathrm{X}$ & $\mathrm{X}$ & $x$ \\
\hline Montreal Cognitive Assessment (MoCA) & & $X$ & & $\mathrm{X}$ & $X$ & $X$ \\
\hline Alcohol Use & & $X$ & & $x$ & $x$ & $X$ \\
\hline
\end{tabular}

Figure 1 The figure depicts the timelines for recruitment, intervention and the collection of study measures.

aged care facilities, whether randomised to the intervention or control arms of the study.

Beyondblue PEAC programme

All care and clinical staff at participating residential aged care facilities will be offered access to the PEAC e-learning programme, an educational package that was developed by Beyondblue that is freely available. Staff are given access to between five and seven modules depending on their professional Background. The modules are: (1) understanding anxiety and depression, (2) anxiety and 
depression in older people, (3) promoting the mental health of community aged care clients, (4) promoting the mental health of aged care residents, (5) identifying and responding to suicide in aged care settings, (6) managing anxiety and depression in aged care clients and residents, and (7) looking after your mental health at work. The programme was designed to address the specific needs of professionals working in the aged care sector, including residential aged care facilities, and can be accessed using an internet browser. Each module takes about $25 \mathrm{~min}$ to complete and successful completion of the programme grants access to a certificate of completion, as well as six Continuing Professional Development hours by the Nursing and Midwifery Board of Australia for registered nursing staff.

An evaluation of the PEAC programme found that the training improved staff knowledge and attitudes about depression, and self-efficacy in responding to residents with depressive symptoms. ${ }^{39}$ Researchers will work alongside managers and Mental Health Champions at participating residential aged care facilities to encourage staff to complete the PEAC, with the aim for completion by at least $50 \%$ of the permanent care and clinical staff during the first 4 weeks of study participation.

\section{Behavioural activation}

The Behavioural Activation intervention will only be undertaken at residential aged care facilities that are randomised to the intervention arm of the trial, although residential aged care facilities in the control arm will be offered the opportunity to participate in a condensed version of the Behavioural Activation training at the end of the study period. Mental Health Champions from intervention residential aged care facilities will take part in a 12 hours Behavioural Activation training course over 2 days with a Behavioural Activation therapist from the research team.

The Behavioural Activation programme is based on the 8-module programme used in the Collaborative cAre for Screem Positive EldeRs (CASPER) trial, where it was delivered by case managers and effectively improved and prevented depressive symptoms in community-dwelling older adults, ${ }^{36} 38$ and has been adapted for use in residential aged care facilities settings for the BAN-Dep trial. Participating residents at each intervention facility will receive a Behavioural Activation manual. The Behavioural Activation manual addresses the following modules: (1) recognition of the symptoms of depression, (2) mood and activity diaries, (3) types of activities (tailored to meet the residents' needs and living environments), (4) breaking jobs down into easier tasks, (5) the benefits of activities, (6) finding ways to be active, (7) spotting symptoms of depression and (8) action plan and activity scheduling.

Mental Health Champions will deliver the intervention with participating residents facilitated by the manual. The intervention is designed so that one module is delivered to the resident each week, apart from modules 7 and 8 , which are designed to be delivered together in one session. It should therefore take 7 weeks to deliver the full programme, but Mental Health Champions will be given the freedom to adapt their delivery to the needs of individual resident participants. For example, each module may be delivered across multiple shorter sessions over the week, or all in one session. It is also recognised that the intervention must accommodate unavoidable breaks such as Mental Health Champions leave or resident illness, so the trial allows up to 12 weeks for Mental Health Champions to complete the modules with participating residents. BAN-Dep investigators trained in Behavioural Activation will also provide up to 1 hour of telephone and/or face-to-face support and supervision sessions to the Mental Health Champions per week.

\section{Strategies to maintain fidelity of the intervention}

Mental Health Champions in the intervention residential aged care facilities will receive a support manual that provides general information about the study, contact details for research team supports, outlines Mental Health Champions responsibilities across each phase of the project and provides specific instructions for delivering individual sessions with participating residents. This will also include examples of effective questions styles and activities. Mental Health Champions will complete fidelity checklists for each contact session, recording coverage of the key components of each module of the intervention as well as any relevant clinical issues that arise. Fidelity checklists will be reviewed during the Mental Health Champions' support sessions.

In recognition of the time that facilities and Mental Health Champions spend on completing study tasks, several reimbursements and incentives were introduced. BAN-Dep will reimburse intervention facilities \$A25 per hour of Behavioural Activation training and support. In addition, Mental Health Champions will receive a $\$ A 15$ gift card for each stage of the intervention that they complete with participating residents, as recorded in the fidelity checklists and support sessions. To incentivise staff completion of the PEAC, in both control and intervention facilities, staff will be asked to place a copy of their completed PEAC certificate in a nominated box at the facility for a prize draw of a $\$ A 50$ gift card to be drawn out at the facility after the 4-week PEAC training window closes.

\section{Management of clinical risk}

Before and after each session, Mental Health Champions will be encouraged to assess any clinical deterioration or risks and report these on the fidelity checklists. Clinically relevant information will be communicated to general practitioners (GPs) and/or other relevant health services. The study will not restrict regular care arranged by the participants' GPs or mental health treating team, including treatment using antidepressants or other psychotherapies. 


\section{Outcome measurements}

\section{Primary outcome measures}

The primary outcome measure of the study will be the PHQ-9, a validated, self-administered depression rating scale comprising of nine items relating to symptoms experienced within the past 2 weeks. ${ }^{42}$ The PHQ-9 is sensitive to changes over time and has shown to be accurate in assessing depressive symptoms in older adults. ${ }^{43}$ Each item is scored on a 4-point scale ranging from 0 (not at all) to 3 (nearly every day). Individual questions assess low mood, loss of interest or pleasure, disrupted sleep, decreased energy, disrupted appetite, feelings of failure or guilt, poor concentration, psychomotor disturbance and suicidal thoughts. PHQ-9 total score can range from 0 (least depressed) to 27 (most depressed). Scores of 10 or more indicate the presence of clinically significant depressive symptoms and will represent the primary outcome of interest of the study. ${ }^{43}$ Changes in the scores of the PHQ-9 between baseline and week 52 will represent a secondary outcome of interest. The scale will be completed by participants during face to face interviews with the study team at baseline, after the intervention is completed (ie, at 3 months), and at 6 and 12 months post-intervention.

\section{Secondary outcomes measures}

Secondary outcomes measures will include the Brief Measure for Assessing Generalised Anxiety Disorder $(\text { GAD-7 })^{45}$ and Montreal Cognitive Assessment ${ }^{46}$ to assess changes in levels of anxiety and cognition over the study period, and the 12-item Short-Form health survey (SF-12), ${ }^{47}$ which provides a valid measure of quality of life for the Australian population. The DeJong Gierveld Loneliness Scale $^{48}$ and Lubben Social Network Scale (LSNS-6) ${ }^{49}$ will be used to measure social connectedness. The Knowledge of Late Life Depression Scale Revised (KLLD-R $)^{50}$ will be used to assess the knowledge of participants and staff about depression. These assessments will be completed by participants during face to face interviews at baseline, 3, 6 and 12 months. Mental Health Champions will self-complete the KLLD-R at the same timepoints.

\section{Other study measures}

Sociodemographic and lifestyle data, including sex, educational achievement, self-reported history of alcohol use and smoking, and prescription of hearing aids or glasses, will be collected from resident participants at baseline. Date of birth and date of admission to the residential aged care facilities will also be collected at baseline from the residents and their medical files. In addition, names, dosages and frequency of use of both regular and PRN (used within the previous 4 weeks) medication, measured height and weight, and clinical diagnoses will be recorded from the residents' clinical files at baseline and at 3, 6 and 12 months. The Modified Barthel Index (m-Barthel) ${ }^{51}$ will be used for the assessment of independence in activities of daily living, and information about falls (frequency and whether they were injurious), unplanned emergency department admissions, and death will also be collected at baseline and 3, 6 and 12 months timepoints.

Sociodemographic information about the Mental Health Champions, including age, gender, education level, length of time they have been working at the residential aged care facilities, and years of work experience in residential aged care facilities will be collected by selfreport at baseline.

\section{Study timeline}

Participating residential aged care facilities will be recruited in waves over the study period, with 6-8 facilities randomised in each wave. Recruitment, consent, screening and baseline assessments of residents, as well as collection of Mental Health Champions measures, will be undertaken by study staff. Staff working at participating residential aged care facilities will be asked to complete the PEAC e-learning package within a 4-week window following the baseline assessment. Mental Health Champions from intervention residential aged care facilities will undertake Behavioural Activation training at the end of this 4-week period.

Mental Health Champions at intervention residential aged care facilities will then have a 12-week timeframe to deliver the 8-module Behavioural Activation intervention described above. At 3, 6 and 12 months, a blinded member of the research team will collect outcome measures from participating residents and Mental Health Champions using questionnaires containing the outcome measures outlined above.

The study is estimated to be completed in 2022. See figure 1 for an outline of the study assessments timeline and figure 2 for the trial recruitment, intervention and assessment steps.

\section{Sample size}

Data from a collaborative community trial delivered in a primary care setting showed that Behavioural Activation decreases conversion to clinically significant depression by $35 \%(95 \%$ CI $9 \%$ to $54 \%)$ over a 12 -month period compared with usual care $(28 \%$ conversion rate in the usual care group).$^{36}$ If a similar effect and conversion rate is present in our study we will require 580 participants (290 in each arm) to declare such a difference as statistically significant between the groups (alpha $=5 \%$, power $=80 \%$ ). It is estimated that the conversion rate to depression in our residential aged care facilities study will be similar to that in the community. As this is a cluster randomised trial, adjustment to the sample size must be made for the design effect (DE). The DE is calculated using the formula: $\mathrm{DE}=1+(\mathrm{n}-1) \rho(\mathrm{n}=$ number of participants per cluster and $\rho=$ intra-class correlation coefficient). Our previous study in residential aged care facilities showed an intra-class correlation coefficient of $0.01 .^{52}$ The expected number of participants per cluster is $6(580 / 100$ residential aged care facilities). Thus, $\mathrm{DE}=1+(6-1) 0.01=1.05$. Hence, this study will require 634 participants (317 per 


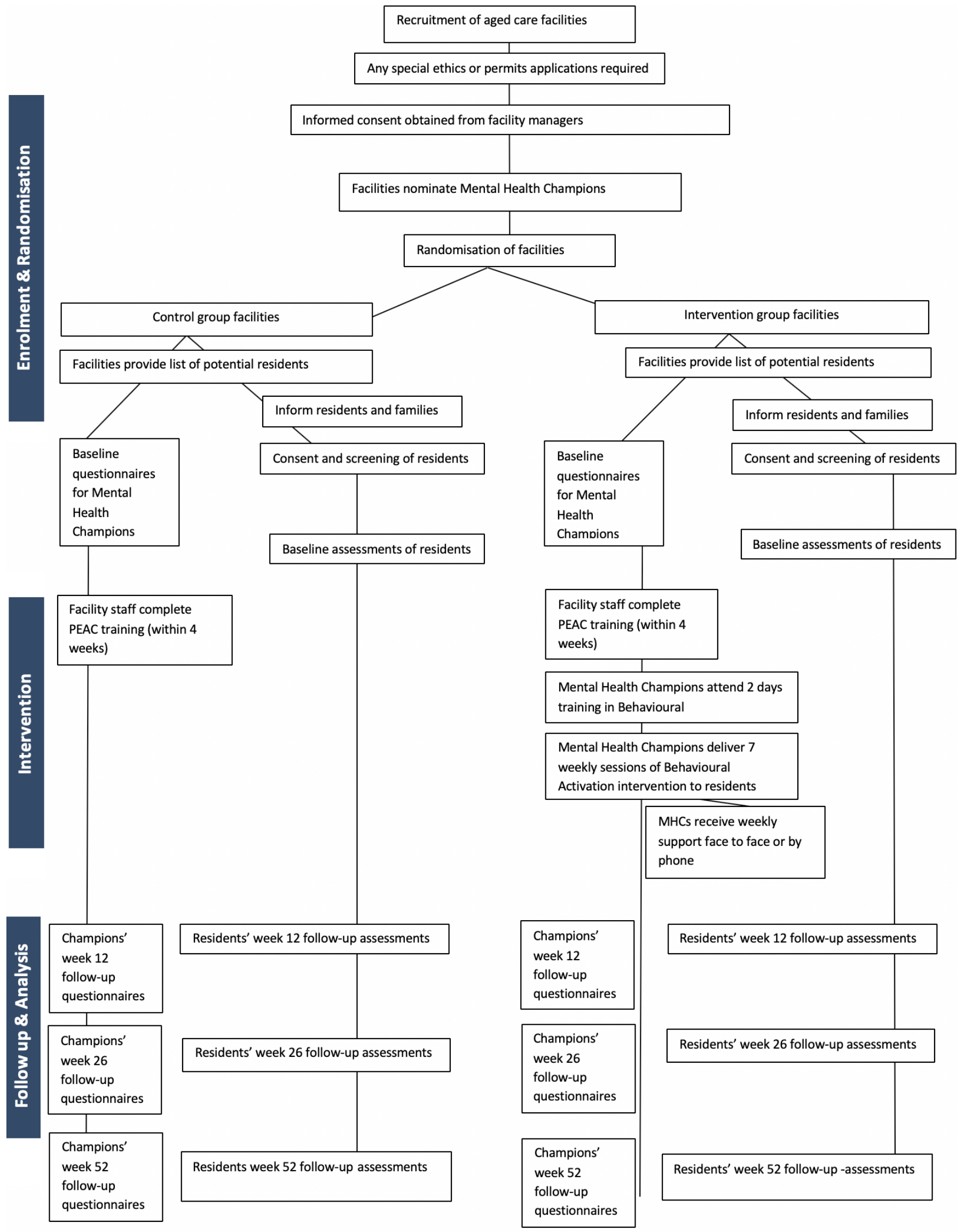

Figure 2 The figure depicts the flow of participants from the time of recruitment, selection and randomisation, to the intervention and collection of study measures. PEAC, Professional Education to Aged Care; MHC, Mental Health Champion. 
group). In addition, it is anticipated that $25 \%$ of participants will be lost during follow-up. ${ }^{8}$ Hence, the study will aim to recruit 666 older adults (333 in each intervention group, about 2-7 per cluster). It is anticipated that 100 residential aged care facilities will need to be recruited in order to meet this sample size of residents.

\section{Recruitment, randomisation, sequence generation, masking and adherence}

The trial will recruit 100 residential aged care facilities located in two Australian cities, Perth and Melbourne (40 residential aged care facilities in Perth and 60 in Melbourne). The participating residential aged care facilities will be randomly assigned to either the Behavioural Activation intervention or control group according to a random list of numbers generated by computer in blocks of 6-8 and stratified by city. The randomisation sequence will be stored in a password-protected server housed at the University of Western Australia and will be managed by a biostatistician not involved in this project. Group assignment will be concealed from residential aged care facilities and research staff. Masking is considered feasible as the duration of the control and active intervention will be similar. Participating residential aged care facilities staff, other than facility managers and Mental Health Champions, will only be advised that the programme aims to test the efficacy of mental health training on the wellbeing of participants.

\section{Blinding}

Research staff involved in the collection of outcomes will remain blind to group assignment for the duration of the trial. Blinded researchers will not be involved in any aspect of the intervention delivery and will be instructed to actively avoid discussing care issues with participants, residential aged care facilities staff, and other research staff. Once the collection of all outcomes is complete, these staff members will be asked to 'guess' the group assignment of residential aged care facilities in order to determine the effectiveness of blinding.

\section{Data collection and management}

Participant information and assessment data, tools and questionnaires will be kept in locked cabinets in a restricted access building and will only be accessible to approved study personnel. Electronic databases of recruitment, assessment and study data will have restricted access and will be password and virus protected, on a network that is protected by firewalls and passwords. The confidentiality of participating facilities, staff and residents will be protected by assigning them a study ID for use on all study materials; identifying information, clinical data and re-identifying information will be stored separately.

The results of this research project will be disseminated through publications and/or presentations in a variety of media to health professionals, academics, clinicians and the public. Only de-identified group data will be presented.

\section{Statistical methods}

All analyses will follow Consolidated Standards of Reporting Trials guidelines. We will use standard descriptive statistics to compare basic sociodemographic and clinical data across treatment arms. The proportion of participants in each study group who present clinically significant symptoms of depression during follow-up (ie, PHQ-9 $\geq 10$ ) will be examined using mixed logit models for the analysis of panel data over 6 and 12 months. Similarly, multilevel mixed models will be used to investigate changes in PHQ-9, GAD-7, SF-12, KLLD-R and m-Barthel over time. Mixed models provide estimates that are 'intention-to-treat' and allow for the investigation of interactions between group and time effects, as well as for the adjustment of possible imbalances between the groups following the randomisation. Imputed chain equations will be employed if loss to follow-up exceeds $25 \%$. Statistical adjustments will be made in the case of unbalanced measures. All probability tests will be two-tailed.

\section{Ethical considerations}

This trial will comply with the principles of the Declaration of Helsinki for Human Rights and will be overseen by the University of Western Australia and Melbourne Health Human Research Ethics Committees, who have approved the study protocol. None of the assessments or procedures are expected, or known, to cause significant harm. Participants will be free to discontinue involvement at any time if they wish.

Written informed consent will be obtained for all participating residential aged care facilities, represented by the facility manager, and verbal consent will be obtained from all staff members who undertake the role of Mental Health Champions. Written informed consent will also be obtained from each resident participating in the trial. As the study is dealing with residents with, or at increased risk of, depression, treating GPs will receive clinically relevant data whenever appropriate. Such a procedure is noted in the participant information and consent form.

\section{Patient and public involvement}

The design of the intervention was based on consultations with older adults in contact with primary health services, which then led to the design of the CASPER trial. This process and the results of the CASPER trial have been described in detail elsewhere. ${ }^{36}{ }^{38}$ We subsequently adapted the intervention for use in aged care facilities after consultation with an aged care provider, Brightwater Care Group, which is represented by Dr Angelita Martini in the team of investigators. Consumers were also involved in providing feedback for the BAN-Dep self-help workbook.

\section{DISCUSSION}

The results of this study will provide important information about whether training local staff members in the use of a structured Behavioural Activation programme 
provides additional benefit above the active control intervention (the Beyondblue PEAC e-learning platform) and decreases the prevalence of depression among older adults living in residential aged care facilities. It is anticipated that if the intervention is shown to be beneficial to residential aged care facilities residents, this knowledge will be transferred into policy and practice through liaison with organisations such as Beyondblue that can incorporate the Behavioural Activation modules into existing educational and intervention packages for residential aged care facilities staff.

The BAN-Dep trial has been designed to be pragmatic, reflecting the real-world environment in which the trial intervention will need to be applied in order to facilitate ease of transition into clinical practice if effectiveness is demonstrated. One strength of this trial design is the introduction of the role of trained Mental Health Champions to ensure that knowledge and skills can be sustained within the residential aged care facilities environment over time through the promotion of mental health upskilling and education and the incorporation of the Behavioural Activation programme into routine care practices. Further strengths of this trial include taking into account the effect of clustering according to residential aged care facilities (older adults living in a certain residential aged care facilities have more in common with each other than with people living in other residential aged care facilities), building on existing resources in residential aged care facilities staff education and the inclusion of cost-effectiveness analysis. Targeting older adults with Major Depressive Disorder as well as those with subthreshold depressive symptoms further allows the study to provide evidence for Behavioural Activation effectiveness across a wider group of residents.

The pragmatic design of this trial does include challenges. The methodology used to assess depressive symptoms means that clinical diagnosis based on established diagnostic criteria (eg, DSM-5) will not be available. The decision to use the PHQ-9 to assess depressive symptoms was based on previous research that has demonstrated that Behavioural Activation interventions are useful for reducing depressive symptoms and preventing the onset of Major Depressive Disorder in older adults with subsyndromal depression. ${ }^{36}$ Moreover, this approach to screening for and assessing depressive symptoms is inexpensive, reliable, valid and can be used routinely in residential aged care facilities, ${ }^{43} 44$ whereas structured clinical interviews are time-intensive and require specialists. Another limitation of this trial is the exclusion of residents with moderate to severe cognitive impairment, which was considered unavoidable due to challenges in obtaining consent from older adults with moderate to severe cognitive impairment. Although this will limit the generalisability of the results to this population, those with mild cognitive impairment who are able to consent will not be excluded from the study and may provide some insight into the ability to deliver Behavioural Activation to those with cognitive difficulties, which could also be compounded by their inability to use the Behavioural Activation workbook. Finally, we have limited our study to residential aged care facilities in metropolitan Perth and Melbourne due to the need for research staff to travel to residential aged care facilities for recruitment, resident assessments and Mental Health Champions support, and for Mental Health Champions to travel to the research offices for Behavioural Activation training. Our findings will therefore benefit from replication targeting residential aged care facilities in diverse geographic, cultural and socioeconomic settings, and perhaps using remote delivery methods.

With limited evidence for and problems associated with antidepressant treatments for older adults in residential aged care facilities, non-pharmacological therapies such as Behavioural Activation can be beneficial for treating and preventing depression in this population. It is hoped that by incorporating Behavioural Activation into routine practice, staff can improve their self-efficacy in responding to depression as well as reduce the prevalence of depression and sustain these improvements over time, leading to significant health and quality of life gains for residents.

In conclusion, this trial will help to develop new knowledge regarding the feasibility and effectiveness of Behavioural Activation interventions in treating symptoms of depression and reducing the overall prevalence of depression in older adults in residential aged care facilities. Reduced depression will, in turn, contribute to lower costs of care and to significant health and quality of life gains for this increasingly prevalent condition among older adults living permanently in aged care facilities.

\section{Trial status}

The trial has been registered with the Australian and New Zealand Trials Registry. Recruitment started in August 2018 and is currently taking place at the time of the submission of this protocol for publication. The trial duration will be from 1 January 2018 to 31 December 2022.

Twitter Simon Gilbody @SimonGilbody

Acknowledgements The authors express their sincere gratitude to the people who have contributed to the development of the intervention, with special thanks to Della Bailey, Seok Lim, Christina Bryant, Kathryn Ellis for all their assistance and guidance.

Contributors Conceived the study: OPA. Designed and obtained funding for the study: OPA, NL, LF, AHF, DLG, CE-B, SG, AM. Setup of database and intervention material: HP, RK, DVR, RL, SMG, DE, EC, TC, NL, DLG, LF, AHF, CE-B, AM, OPA. Training and supervision of staff: DE, HP, KAE, EC, TC, NL, OPA. Recruitment: HP, RK, OPA, CE-B, AM, DVR, RL, DLG, EC, TC, KAE, NL. Data collection: DVR, RL, HP and RK. Drafting of the manuscript: DVR led the drafting of the manuscript with the support and important intellectual input from all authors. Final approval of the manuscript version to be published: all authors.

Funding This study is supported by a grant from the National Health and Medical Research Council (NHMRC) and Beyondblue. The funding source had no role in any part of the design of this trial. It will also have no part in the execution, data collection, analysis or involvement in decision-making of the trial. 
Competing interests None declared.

Patient consent for publication Not required.

Ethics approval The trial will comply with the principles of the Declaration of Helsinki for Human Rights and will be overseen by the University of Western Australia (reference RA/4/20/4234) and Melbourne Health (reference number HREC/18/MH/47) Human Research Ethics Committees, who have approved the trial. Written informed structured consent will be required from all participants. None of the assessments or procedures are expected, or known, to cause significant harm, and participants will be free to discontinue involvement if they wish. As the study is dealing with a population with, or at increased risk of, depression, treating GPs will receive clinically relevant data. We will also ensure referral to the relevant services to anyone identified to be a significant risk of self-harm.

Provenance and peer review Not commissioned; externally peer reviewed.

Open access This is an open access article distributed in accordance with the Creative Commons Attribution Non Commercial (CC BY-NC 4.0) license, which permits others to distribute, remix, adapt, build upon this work non-commercially, and license their derivative works on different terms, provided the original work is properly cited, appropriate credit is given, any changes made indicated, and the use is non-commercial. See: http://creativecommons.org/licenses/by-nc/4.0/.

ORCID iDs

Terence Chong http://orcid.org/0000-0003-2432-8193

Osvaldo P Almeida http://orcid.org/0000-0002-8689-6199

\section{REFERENCES}

1 Lim GY, Tam WW, Lu Y, et al. Prevalence of depression in the community from 30 countries between 1994 and 2014. Sci Rep 2018;8:2861.

2 Pirkis J, Pfaff J, Williamson M, et al. The community prevalence of depression in older Australians. J Affect Disord 2009;115:54-61.

3 Magnil M, Janmarker L, Gunnarsson R, et al. Course, risk factors, and prognostic factors in elderly primary care patients with mild depression: a two-year observational study. Scand J Prim Health Care 2013;31:20-5.

4 Almeida OP, Hankey GJ, Yeap BB, et al. Depression, frailty, and all-cause mortality: a cohort study of men older than 75 years. J Am Med Dir Assoc 2015:16:296-300.

5 Soysal P, Veronese N, Thompson T, et al. Relationship between depression and frailty in older adults: a systematic review and metaanalysis. Ageing Res Rev 2017;36:78-87.

6 Almeida OP, Alfonso H, Pirkis J, et al. A practical approach to assess depression risk and to guide risk reduction strategies in later life. Int Psychogeriatr 2011;23:280-91.

7 Seitz D, Purandare N, Conn D. Prevalence of psychiatric disorders among older adults in long-term care homes: a systematic review. Int Psychogeriatr 2010;22:1025-39.

8 Kotynia-English $\mathrm{R}$, McGowan $\mathrm{H}$, Almeida OP. A randomized trial of early psychiatric intervention in residential care: impact on health outcomes. Int Psychogeriatr 2005;17:475-85.

9 Avasthi A, Grover S. Clinical practice guidelines for management of depression in elderly. Indian J Psychiatry 2018;60:S341-62.

10 McCabe MP, Karantzas GC, Mrkic D, et al. A randomized control trial to evaluate the beyondblue depression training program: does it lead to better recognition of depression? Int J Geriatr Psychiatry 2013;28:221-6.

11 Frost R, Beattie A, Bhanu C, et al. Management of depression and referral of older people to psychological therapies: a systematic review of qualitative studies. Br J Gen Pract 2019;69:e171-81.

12 Kok RM, Reynolds CF. Management of depression in older adults. JAMA 2017;317:2114-22.

13 The Royal Australian College of General Practitioners - 'Silver Book' National Taskforce. Medical care of older persons in residential aged care facilities. 4th edition, 2006.

14 Rodda J, Walker Z, Carter J. Depression in older adults. BMJ 2011;343:d5219-7

15 Hanlon JT, Handler SM, Castle NG. Antidepressant prescribing in US nursing homes between 1996 and 2006 and its relationship to staffing patterns and use of other psychotropic medications. J Am Med Dir Assoc 2010;11:320-4.

16 Westbury J, Gee P, Ling T, et al. More action needed: psychotropic prescribing in Australian residential aged care. Aust N Z J Psychiatry 2019;53:136-47.

17 Banerjee S, Hellier J, Dewey M, et al. Sertraline or mirtazapine for depression in dementia (HTA-SADD): a randomised, multicentre, double-blind, placebo-controlled trial. The Lancet 2011;378:403-11.
18 Ford AH, Almeida OP. Management of depression in patients with dementia: is pharmacological treatment justified? Drugs Aging 2017;34:89-95

19 Dudas R, Malouf R, McCleery J, et al. Antidepressants for treating depression in dementia. Cochrane Database Syst Rev 2018;2.

20 Boyce RD, Hanlon JT, Karp JF, et al. A review of the effectiveness of antidepressant medications for depressed nursing home residents. $J$ Am Med Dir Assoc 2012;13:326-31.

21 Dörks M, Schmiemann G, Hoffmann F. Pro re NatA (as needed) medication in nursing homes: the longer you stay, the more you get? Eur J Clin Pharmacol 2016;72:995-1001.

22 Farina N, Morrell L, Banerjee S. What is the therapeutic value of antidepressants in dementia? A narrative review 2017:32-49.

23 Park H, Satoh H, Miki A, et al. Medications associated with falls in older people: systematic review of publications from a recent 5-year period. Eur J Clin Pharmacol 2015;71:1429-40.

24 Ekers D, Webster L, Van Straten A, et al. Behavioural activation for depression; an update of meta-analysis of effectiveness and sub group analysis. PLoS One 2014;9:11.

25 Davison TE, You E, Doyle C, et al. Psychological therapies for depression in older adults residing in long-term care settings. Cochrane Database Syst Rev 2018;8.

26 Richards DA, Ekers D, McMillan D, et al. Cost and outcome of behavioural activation versus cognitive behavioural therapy for depression (cobra): a randomised, controlled, non-inferiority trial. The Lancet 2016;388:871-80.

27 Shinohara K, Honyashiki M, Imai H, et al. Behavioural therapies versus other psychological therapies for depression. 2013;138.

28 Cuijpers P, van Straten A, Warmerdam L. Behavioral activation treatments of depression: a meta-analysis. Clin Psychol Rev 2007;27:318-26.

29 Dimidjian S, Hollon SD, Dobson KS, et al. Randomized trial of behavioral activation, cognitive therapy, and antidepressant medication in the acute treatment of adults with major depression. $J$ Consult Clin Psychol 2006;74:658-70.

30 Kanter JW, Manos RC, Bowe WM, et al. What is behavioral activation?A review of the empirical literature. Clin Psychol Rev 2010;30:608-20.

31 Ferster CB. A functional anlysis of depression. Am Psychol 1973;28:857-70.

32 Markle-Reid M, McAiney C, Forbes D, et al. An interprofessional nurse-led mental health promotion intervention for older home care clients with depressive symptoms. BMC Geriatr 2014;14:62.

33 Leontjevas R, Gerritsen DL, Smalbrugge M, et al. A structural multidisciplinary approach to depression management in nursinghome residents: a multicentre, stepped-wedge cluster-randomised trial. The Lancet 2013;381:2255-64.

34 Meeks S, Van Haitsma K, Schoenbachler B, et al. BE-ACTIV for depression in nursing homes: primary outcomes of a randomized clinical trial. J Gerontol 2015;70:13-23.

35 Orgeta V, Brede J, Livingston G. Behavioural activation for depression in older people: systematic review and meta-analysis. $\mathrm{Br}$ J Psychiatry 2017;211:274-9.

36 Gilbody S, Lewis H, Adamson J, et al. Effect of collaborative care vs usual care on depressive symptoms in older adults with subthreshold depression. JAMA 2017;317:728-37.

37 Beer C, Lowry R, Horner B, et al. Development and evaluation of an educational intervention for general practitioners and staff caring for people with dementia living in residential facilities. Int Psychogeriatric 2011:23:221-9.

38 Pasterfield M, Bailey D, Hems D, et al. Adapting manualized behavioural activation treatment for older adults with depression. Cogn Behav Therap 2014;7:1-11.

39 Mellor D, Kiehne M, McCabe MP, et al. An evaluation of the beyondblue Depression Training Program for aged care workers. Int Psychogeriatr 2010;22:927-37.

40 Whooley MA, Avins AL, Miranda J, et al. Case-Finding instruments for depression. two questions are as good as many. J Gen Intern Med 1997;12:439-45.

41 Folstein MF, Folstein SE, McHugh PRAlbanese MA, Ward SB, eds. Mini-Mental state examination, 1975.

42 Kroenke K, Spitzer RL, Williams JB. The PHQ-9: validity of a brie depression severity measure. J Gen Intern Med 2001;16:606-13.

43 Löwe B, Kroenke K, Herzog W, et al. Measuring depression outcome with a brief self-report instrument: sensitivity to change of the patient health questionnaire (PHQ-9). J Affect Disord 2004;81:61-6.

44 Phelan E, Williams B, Meeker K, et al. A study of the diagnostic accuracy of the $\mathrm{PHQ}-9$ in primary care elderly. BMC Fam Pract 2010;11:63. 
45 Spitzer RL, Kroenke K, Williams JBW, et al. A brief measure for assessing generalized anxiety disorder. Arch Intern Med 2006;166:1092-7.

46 Nasreddine ZS, Phillips NA, Bédirian V, et al. The Montreal cognitive assessment, MoCA: a brief screening tool for mild cognitive impairment. J Am Geriatr Soc 2005;53:695-9.

47 Sanderson K, Andrews G, Sanderson K. The SF-12 in the Australian population: cross-validation of item selection. Aust N Z J Public Health 2002;26:343-5.

48 Gierveld JDJ, Tilburg TV, De Jong Gierveld J. A 6-Item scale for overall, emotional, and social loneliness. Res Aging 2006;28:582-98.
49 Lubben JE. Assessing social networks among elderly populations. Fam Community Health 1988;11:42-52.

50 Karantzas GC, Davison TE, McCabe MP, et al. Measuring carers' knowledge of depression in aged care settings: the knowledge of late life depression Scale-Revised. J Affect Disord 2012;138:417-24.

51 Mahoney FI, Barthel DW. Functional evaluation: the Barthel index: a simple index of independence useful in scoring improvement in the rehabilitation of the chronically ill. Maryland State Med J 1965;14:61-5.

52 Beer C, Horner B, Flicker L, et al. A cluster-randomised trial of staff education to improve the quality of life of people with dementia living in residential care: the direct study. PLoS One 2011;6:e28155-11. 